\section{Ein langer Weg bis zu neuen Standards für Weiterbildung in der EU}

\author{
Fachgesellschaften diskutieren derzeit neue Mindeststandards \\ für die Weiterbildung zum Facharzt in der EU. Darüber ent- \\ scheiden würden am Ende aber die zuständigen Stellen in den \\ Mitgliedsländern oder die politischen EU-Gremien.
}

Geboten wird ein „European Curriculum“ - nicht weniger als ein neuer Mindeststandard für die Ausbildung zum Orthopäden und Unfallchirurgen in der Europäischen Union. Seit Januar 2015 kursiert das Papier, erarbeitet im Umfeld von EFORT. Mehr wie einige Stichworte wurden bis Drucklegung dieser Ausgabe der ZFOU nicht verraten, aber immerhin: Das Papier trage eine stark angelsächsische Handschrift und sei eine akzeptable Diskussionsgrundlage, erklärt der ehemalige Generalsekretär der DGOU Fritz Uwe Niethard im ZFOU-Interview (siehe [1] und das Interview ab Seite 235).

Schon bald also neue Anforderungen für alle, die hierzulande den Facharzt in Orthopädie und Unfallchirurgie machen möchten? Eine einheitliche Facharztausbildung für ganz Europa?

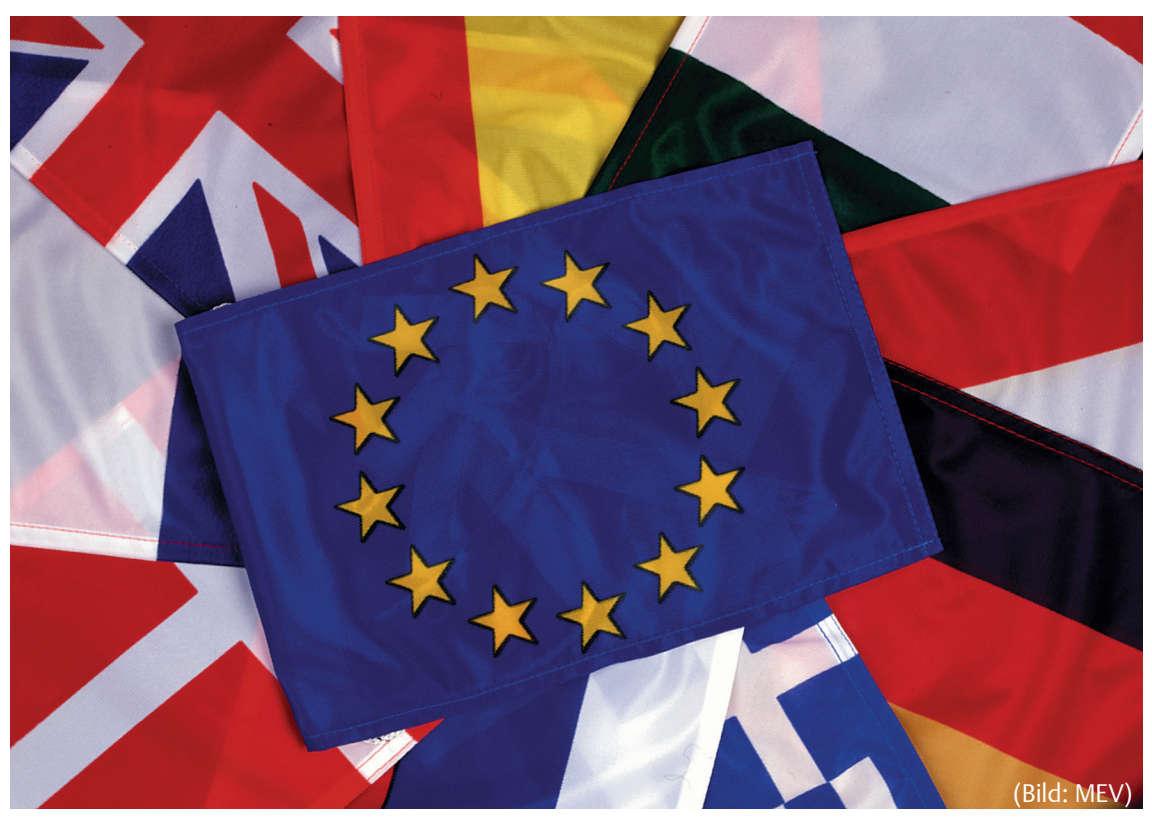

Mitnichten. Zunächst mal müssten sich die Experten untereinander einigen. Der EFORT-Vorlage zustimmen sollte ursprünglich schon im Mai 2015 eine Unterabteilung der UEMS, die Sektion Orthopädie und Traumatologie. Daraus wurde nichts. „Das Papier ist wieder bei EFORT, die müssen nachbessern“, weiß einer der beiden deutschen Vertreter in diesem Gremium der UEMS, Joachim Grifka, Chefarzt am Asklepios Klinikum Bad Abbach. Vermutlich werde man auf der Herbsttagung der Sektion abstimmen. Bis dann wirklich Vorschläge für neue Standards der Weiterbildung in Europa womöglich gar Auswirkungen auf die hiesige Szene haben - „das dauert noch Jahre“, erklärt Grifka. Da müssten dann erst nationale Gremien zustimmen - in Deutschland am Ende der Ärztetag und die 17 Ärztekammern. Thema beendet.
Nicht ganz. Eine von Fachgesellschaften zunächst in Hinterzimmern geführte Debatte um neue Standards in der EU schafft womöglich den Humus, aus dem vermeintlich urplötzlich doch neue und offizielle Spielregeln erwachsen. Unwissenheit schützt dann vor neuer Regelung nicht. Und Experten wie Niethard monieren eine sträfliche Abstinenz. Die Zahl deutscher Vertreter in den EU-Fachgremien sei deutlich ausbaufähig, erklärt Niethard, bis heute beratend bei der EFORT dabei: „Wir brauchen das Engagement von mehr jungen Leuten“ (siehe Interview Niethard, Seite 235).

Gremienarbeit in der EU? EFORT? UEM... was? Europa lernen heißt Abkürzungen lernen.

EFORT ist Treffpunkt der europäischen Orthopäden und Unfallchirurgen - Zusammenschluss der nationalen Fachgesellschaften, zum Teil auch Berufsverbänden auf diesem Gebiet (siehe auch Kasten EFORT Seite 232). Die EFORT hat die aktuelle Diskussion um neue Standards für die Weiterbildung für $\mathrm{O}$ und $\mathrm{U}$ in Europa angeschoben, seit 2012 schon kursieren Diskussionsvorlagen.

UEMS ist größeres Kaliber. Hier treffen sich auf EU-Ebene die nationalen Fachgesellschaften aus allen medizinischen Fachgebieten. Straff organisiert kommt sie daher, hat „Sektionen“ und „Divisionen“. Die leicht militärisch gefärbte Terminologie ist Nein, kein negativer deutscher Einfluss in Europa. UEMS steht in reinstem Französisch für Union Européenne des Médicins Spécialistes. Man sieht sich als das Spreachrohr für den Facharzt aller Richtungen auf internationalem Parkett. Die UEMS ist eine besonders gewichtige Stimme auch bei der Lobbyarbeit der Ärzte gegenüber den politischen EU-Gremien.

Gegründet wurde die UEMS am 20. Juli 1958, von Fachgesellschaften aus 6 Ländern. Heute kommen sie aus 28 EU-Ländern, aus Norwegen, der Schweiz, aus weiteren assoziierten Ländern wie Armenien, Israel, der Türkei. 1,6 Millionen Fachärzte vertritt die UEMS nach eigenen Angaben. Mitglieder sind aber allein die Fachgesellschaften, eine individuelle Mitgliedschaft gibt es nicht.

Auch die Zahl der Untergliederungen hat seit 1958 rasant zugenommen. 41 Sektionen gibt es heute, jede steht für ein Großgebiet der Medizin [2]. 


\section{EFORT und UEMS}

EFORT ist die European Federation of National Associations of Orthopaedics and Traumatology [9]. Die 1991 gegründete Organisation vereinigt aktuell 45 Fachgesellschaften aus 41 Ländern - vorrangig Fachgesellschaften der Orthopäden aus Europa, aber auch Unfallchirurgen und einige Berufsverbände. Aus Deutschland sind so nicht nur die DGOOC, auch die DGU und der BVOU Mitglied. Als assoziierte Mitglieder sind orthopädische Gesellschaften aus Armenien, aus Israel, Ägypten, Jordanien unter anderem mit dabei, als „Specialty Societies“ etwa auch die brasilianische Fachgesellschaft SBOT.

Hauptaktivität ist der Austausch der Fachgesellschaften international, dafür gibt es eine eigene Jahrestagung, den EFORT-Kongress (der letzte war vom 27. bis 29. Mai in Prag), spezielle Fachforen wie das Expertentreffen ExMEx, Journals, und jungen Ärzten winken auch Stipendien. Es war die EFORT, die schon 2012 einen Entwurf für neue Standards in der Weiterbildung für Orthopäden in der EU vorlegte - ein Papier, das allerdings bis heute im Diskussionsstadium steckt.

UEMS steht für Union Européenne des Médicins Spécialistes [10]. Es ist das Gremium, in dem sich auf EU-Ebene die nationalen Fachgesellschaften aus allen medizinischen Fachgebieten treffen. Die Zustimmung der UEMS zu neuen Minimalstandards für die Weiterbildung in allen Ländern der EU gilt als entscheidend, um das Thema womöglich auch bei den politischen Gremien der EU auf die Agenda zu hieven.

\section{UEMS-Prüfungen}

Am ehesten geläufig wird die Organisation jenen Ärztinnen und Ärzten sein, die eigene UEMS-Examina ablegen. Rein freiwillig, wie etwa das EBSQ-Examen - ein „Siegel für Exzellenz“, wie es Hans-Jörg Oestern, Leiter einer Division Traumatologie in der UEMS, formuliert.

EBS... was? EBSQ steht für European Board of Surgery Qualification. Das Examen ist eine Qualifikation der Sektion für Chirurgie. Zu haben in 7 verschiedenen chirurgischen Schwerpunkten, „Divisionen“, etwa in Allgemeinchirurgie, Brustchirurgie oder Unfallchirurgie / Traumatologie [3].
Orthopäden und Unfallchirurgen wiederum sind in der UEMS nicht bei den Chirurgen sortiert, haben vielmehr ihre eigene Sektion für Orthopädie und Unfallchirurgie (Section of Orthopaedics and Traumatology [4]) nebst eigenem Board-Examen: Das EBOT-Examen des European Board of Orthopaedics and Traumatology [5].

Die Teilnehmerzahlen bei den Prüfungen, die in der Regel jährlich absolviert werden können, sind nicht hoch. Maximal 300 Ärzte hatten Ende 2014 das EBOT-Examen, das es seit 2001 gibt, in der Tasche. Wer einen schriftlichen Teil bestanden hat (Multiple Choice), kann sich der mündlichen Prüfung stellen. Beim EBOT-Examen wechseln die Prüfungsorte von Jahr zu Jahr und sogar zwischen 1. und 2. Teil. Das EBSQ-Examen der Division Traumatologie bei den Chirurgen findet hingegen jährlich beim DKOU in Berlin statt. Es richtet sich an Unfallchirurgen, mit Schwerpunkt Knochen und Muskeln, aber etwa auch an Viszeralchirurgen. Bis zu einem Dutzend Teilnehmer belegen aktuell dieses Examen Jahr für Jahr.

Die Kriterien der Prüfungen gelten als streng. 65 von 100 Teilnehmern am EBOTExamen 2013 kamen durch den schriftlichen Teil. Von ihnen schaffte $74 \%$ den folgenden mündlichen Teil [6]. 2014 machten 109 Ärzte beim schriftlichen Teil und noch 82 beim mündlichen Teil mit, die Erfolgsquote lag bei $72 \%$. „Die Prüfung macht man zur eigenen Qualifikation“, betont Joachim Grifka, zuständig für die Auswahl der bundesdeutschen Teilnehmer beim EBOT-Examen.

\section{Kein Ersatz für Facharztprüfung}

1984 hob die UEMS das erste europäische Examen aus der Tasche, mittlerweile gibt es sie für rund 30 Disziplinen. Irreführend ist der gelegentlich zu lesende Terminus vom „EU-Facharztexamen“. Genau das sind diese Examen bislang eben nicht. Es gibt keine Facharztprüfungen auf EU-Ebene. Den Facharzttitel gibt es nur nach den Spielregeln im jeweiligen EU-Land. Mehr noch: Nur wer bereits einen Facharzttitel nebst Wohnsitz in einem EU-Land hat, kann sich für EBOT oder EBSQ-Examen bewerben.

Dabei hat die UEMS mit diesen Examina durchaus Größeres vor. Die Harmonisierung der Weiterbildung ist von Anfang an Hauptziel der Organisation. Das Ziel von
Prüfungen wie dem EBOT-Examen sei es, einen qualitativ hochwertigen und vergleichbaren Stand bei der Qualifikation von medizinischen Spezialisten in der EU sicher zu stellen, betont eine Gruppe um Tatu J. Mäkinen von der Helsinki University, die 2013 die Zahlen zum EBOT-Examen unter die Lupe nahm. Und das EBOT-Examen könnte durchaus dieser Standard in der Orthopädie sein, so Tenor des Papers, könnte als Benchmark dienen. Das allerdings ist ein Wunsch, der bislang kaum in Erfüllung ging.

Die Unterschiede der Gesundheitssysteme in den Ländern der EU bleiben groß, damit auch die Wege zum Facharztberuf. Schon der bloße Blick auf den Umfang der Ausbildungsordnungen zum Facharzt für Orthopädie [1], lässt gewaltige Unterschiede erahnen. 212 Seiten hat das britische Curriculum, die Portugiesen umreißen ihre Vorgaben auf 6 Druckseiten, die Italiener haben eine Seite mehr. In manchen Ländern, allen voran Großbritannien, kümmern sich die Fachgesellschaften und Berufsverbände um Inhalte und Prüfungsabnahmen, in Frankreich machen es die Unis in weitgehender Autonomie, wieder in anderen, allen voran in Deutschland, haben die Ärztekammern große Autonomie. Eine einheitliche Weiterbildung zum Facharzt nebst Prüfungsordnung ist in der EU weit weg.

Und, Verwirrung komplett - sie ist laut Gesetz gar nicht mehr nötig. Denn im Zuge der Harmonisierung hat die EU längst dafür gesorgt, dass alle Mitgliedsländer untereinander ihre jeweiligen Prüfungen und Facharzttitel gegenseitig anerkennen. Ein bayerischer Orthopäde ist so gut wie sein portugiesischer Kollege.

\section{Europa, Richtlinie 2005/36/EG $\nabla$}

Es ist die nur logische Konsequenz aus dem EU-Grundrecht auf Freizügigkeit, jeder EU-Bürger kann seinen Beruf in allen Mitgliedsländern der EU ausüben. Die EURichtlinie über die Anerkennung von Berufsqualifikationen vom 07. September 2005 (Richtlinie 2005/36/EG) zählt Ärzte, Zahnärzte wie Krankenpfleger zu den ,reglementierten“ Berufen, bei denen die Mitgliedsstaaten bestimmte, in einem Anhang aufgeführte, Berufsabschlüsse gegenseitig anerkennen.

Anhang 5.1.1 der Richtlinie listet alle Stellen aus den Mitgliedsländern auf, deren 
Arzt- und Facharzttitel gleichrangig zur ärztlichen Berufsausübung in der EU berechtigen. In Deutschland ist es die von den Landesbehörden verliehene Approbation. In Frankreich oder Portugal sind es die Unis, die ein staatliches Diplom zum Mediziner vergeben, in Großbritannien sind es die „competent examining bodies“ - oft die Fachgesellschaften, die Prüfungen abnehmen. Als Mindeststandard für die Medizinerausbildung nennt die Richtlinie mindestens 6 Jahre, alias 5500 Stunden „theoretischen und praktischen Unterrichts“, abzuleisten „an einer Universität oder unter Aufsicht einer Universität“", bevor sich jemand nach Prüfungen Arzt nennen kann.

Der Ansatz der Richtlinie ist der einer Mindestharmonisierung, erläutert die Pressestelle der EU-Kommission in Berlin. Folglich könne jeder Mitgliedstaat auf nationaler Ebene zwar gerne höhere Anforderungen vorschreiben, müsse aber Berufsinhaber anerkennen, die ihre Qualifikationen in anderen Mitgliedstaaten erworben haben, sofern diese den Mindestanforderungen entsprechen. Die UEMS ist, nebenbei, einer von vielen Ärzteverbänden, die beratend diese Spielregeln mitentwickelt haben.

Der gleiche Ansatz gilt bei den Weiterbildungen zum Facharzt. Derzeit hat die Richtlinie Vorgaben zu 54 „Kategorien von Facharztberufen“. Anhang 5.1.3. nennt Fachgebiete und Mindestdauer der Weiterbildungen. Für Orthopädie sind es 5 Jahre - das liegt ein Jahr unter dem Durchschnitt der hiesigen Weiterbildung in $\mathrm{O}$ und U. Und wieder zeichnet die Liste der autorisierten Stellen den Pluralismus der Ausbildungswege in Europa nach. In Deutschland sind es die Landesärztekammern, die den Titel Facharzt vergeben, in Frankreich können es von Universitäten verliehene Facharzttitel sein, aber auch solche des Conseil de l'Ordre des Médicins. Zu Mindestanforderungen an Inhalte der Weiterbildungen sagt die Richtlinie nichts.

\section{Verfahren hat sich bewährt \\ $\nabla$}

In Deutschland setzt seit April 2012 ein Anerkennungsgesetz die EU-Vorgaben um - die Summe aus einem Bundesgesetz, dem Berufsqualifikationsfeststellungsgesetz und rund 60 weiteren Regelungen $\mathrm{zu}$ Berufsqualifikationen - etwa der Bundesärzteordnung.
Der Tenor eines umfangreichen Leseangebots zum Thema von Bundesregierung, Ärztekammern oder Marburger Bund aus dem Internet (siehe auch die Links am Ende des Textes): Eine Mediziner- und Facharztweiterbildung aus einem anderen EU-Land bedarf keiner ,individuellen Gleichwertigkeitsprüfung“, sondern wird automatisch anerkannt, wenn sie der Richtlinie 2005/36/EG entspricht. Zuständig für die Anerkennung eines im Ausland erworbenen Arzttitels für eine Approbation sind bei uns die Behörden im jeweiligen Bundesland, für die Anerkennung eines Facharzttitels sind es die Ärztekammern.

Die Statistiken deuten es an: Deutschland ist für Ärzte aus anderen Ländern nicht unattraktiv. 13344 Anträge auf Anerkennung einer beruflichen Qualifikation von Bürgern aus anderen EU-Ländern beschieden die Behörden danach allein im Jahr 2013. Das Gros kam von Ärzten (6687) und Krankenpflegern (3810). Abgelehnt wurden gerade mal $4 \%$ aller Fälle [7].

Nach der Statistik der Bundesärztekammer kamen allein 20143768 Ärzte neu nach Deutschland, knapp die Hälfte, 1692, aus Ländern der EU.

Insgesamt zählte die BÄK Ende 2014 39661 Ärztinnen und Ärzte im Bundesgebiet, die ursprünglich aus anderen Ländern kamen, darunter über die Hälfte (22080) aus EU-Ländern. 9,5\% aller 365247 Ärzte, die insgesamt im Bundesgebiet arbeiten, kommen aus anderen Ländern.

Die Zahl der Kolleginnen aus dem Ausland hat sich seit 2000 fast vervierfacht. Im Vergleich der EU-Länder stammen besonders viele Ärztinnen und Ärzte aus Rumänien (3857) und Griechenland (3011), gefolgt von Österreich (2695). Relativ hohe Zahlen auch aus 2 Nicht-EU-Ländern: Knapp 2000 Ärzte sind aus Russland, fast 1100 aus der Ukraine.

Gerade in ländlichen Regionen leisteten die Ärzte aus dem Ausland einen wichtigen Beitrag zur Aufrechterhaltung der medizinischen Versorgung, betonte BÄKChef Frank- Ulrich Montgomery bei der Vorlage der Ärztestatistik 2014.

Verschärft worden sind die Ansprüche an die Sprachkenntnisse. 2014 haben die Gesundheitsminister der Länder festgelegt, dass Ärzte, Krankenpfleger oder Psycholo-
Weitere Ärzteorganisationen in der EU

UEMS ist nicht das einzige Ärztegremium in der EU. Mit zu den größten, bei auch inhaltlichen Überscheidungen, zählen:

CPME, Comité Permanent des Medicins Européenes [11], alias Standing Committee of European Doctors. Gremium, Zusammenschluss nationaler Standesorganisationen, Ärztekammern. Deutsches Mitglied ist die Bundesärztekammer. Standards für die Qualifikation ist dito Schwerpunkt.

UEMO, European Union of General Practitioners [12], ein Zusammenschluss von Fachgesellschaften in Allgemeinmedizin

AEMH, European Association of Senior Hospital Physicians [13], Zusammenschluss von leitenden Krankenhausärzten. Hier ist eine persönliche Mitgliedschaft möglich.

gen ein „allgemeines Sprachniveau“ der Stufe B2 schaffen, und ein Fachsprachenniveau der Stufe C1 nachweisen müssen. Zuständig für die Sprachprüfung sind die Approbationsstellen im Bundesland. Einige haben dies den Kammern übertragen, darunter sind Westfalen-Lippe, Rheinland-Pfalz, Sachsen-Anhalt. Und immer mehr Kammern, Krankenhäuser und Ärztenetze helfen beim Einfinden in die neue Umgebung, bieten Sprach- und Integrationskurse an.

Die Integration der Ärzte aus dem Ausland funktioniere gar nicht so schlecht, erklärte Reiner Felsberg, Geschäftsführer beim Marburger Bund Berlin-Brandenburg Mitte April 2015 gegenüber dem Deutschen Ärzteblatt. So richtig gut ist sie mancherorts aber noch nicht. Es sei wichtig, so Felsberg, die ausländischen Ärzte nicht einfach nur als Lückenbüßer zu sehen. Nötig sei eine neue Willkommenskultur, zu zeigen, dass man sich über die neuen Kollegen freue und wünsche, dass sie auch auf Dauer blieben.

Unklar bleibt derweil, wie viele Fachärzte aus EU-Ländern zu uns kommen, eine offizielle Statistik dazu gibt es nicht - auch wenn die Zahlen bei den Ärztekammern liegen müssten.

Dieses „operative Geschäft“ laufe bei den Bezirksärztekammern, so die Auskunft 
bei der Ärztekammer Baden-Württemberg in Stuttgart, eine Landesstatistik gebe es nicht. Die Spielregeln seien klar: „Legt ein Antragsteller Unterlagen vor, nach denen er eine Weiterbildung im Ausland absolviert hat, die in den Anhängen der EU-Richtlinie aufgeführt sind, dann muss ihm die Anerkennung gewährt werden“, so die Auskunft aus Stuttgart. Zu Qualitätsunterschieden, gar -problemen angesichts womöglich stark abweichender Weiterbildungswege je nach EU-Land keine Info.

Gar kein Statement zum Thema gibt es bei der Pressestelle der Bayerischen Ärztekammer. Mehr Infos hingegen aus Mainz. „EU-Recht ist für uns bindend“ betont auch Jürgen Hoffart, Geschäftsführer der Ärztekammer Rheinland-Pfalz. „Sobald es sich um einen in einem anderen EULand erworbenen Facharzt handelt, der von der Berufsanerkennungsrichtlinie aufgeführt wird, wird das von uns anerkannt, wir müssen das anerkennen.“ Und ja, es gebe durchaus qualitative Unterschiede im Vergleich der Ausbildungen in verschiedenen Ländern. So seien in Frankreich nur 3 Jahre bis zur Vergabe des Titels Allgemeinmediziner nötig, in Deutschland 5. Dennoch sieht Hoffart keine gravierenden Probleme mit möglichen Qualitätsunterschieden. Dafür sei allein schon die Zahl der Ärzte, die als Fachärzte nach Deutschland kommen, zu klein. Hoffart: „Wir schreiben etwa in der Bezirksärztekammer Rheinhessen 4 Fälle im Jahr um, $98 \%$ aller Ärzte kommen mit der Approbation oder der Grundausbildung gerade deshalb zu uns, weil sie auf der Suche nach einer guten Weiterbildungsmöglichkeit sind.“

\section{Keine Harmonisierung der Ge- sundheitssysteme aus Brüssel $\nabla$}

Zufrieden mit dem Prozedere äußert sich die Pressestelle der EU-Kommission. Das System der Richtlinie 2005/36/EG habe sich bei der Anerkennung von Facharztqualifikationen als effektiv erwiesen und man habe keine Informationen über „diesbezügliche Probleme“ erhalten. Bei öffentlichen Anhörungen im Rahmen einer Novellierung der Richtlinie im Jahr 2011 sei es überwiegende Auffassung gewesen, dass die Richtlinie weiterhin keine Vorgaben über Tätigkeiten, Kenntnisse und Fähigkeiten für Facharzttätigkeiten macht. Dabei soll es für Brüssel offenbar bleiben: „Das Gesundheitswesen liegt in der Kompetenz der Mitgliedstaaten und es gibt keine Pläne für eine EU-weite Harmonisierung in absehbarer Zeit“, erläutert die Kommissions-Pressestelle zur Frage, ob in Brüssel an neuen Mindeststandards etwa für die Weiterbildung zum Facharzt getüftelt wird.

Auf der anderen Seite grummelt die aktuelle Debatte in der Fachwelt eben um mehr Definitionen für die Inhalte der Weiterbildung. „Wir haben zwar die Mimimalanforderungen in der EU-Richtlinie, aber die sind überholt und müssen überarbeitet werden“, betont Joachim Grifka.

Wenn eine Einigung bei der UEMS gelingt, frühester Termin sind die Sektionstreffen der UEMS im Herbst 2015, ginge ein Vorschlag am Ende von der UEMS auch an die EU-Kommission. Fernziel: Neue Minimalstandards für die EU-Richtlinie zur Berufsqualifikation.

\section{Board-Examina der UEMS \\ $\nabla$}

Und wenn nicht? Neue Standards könnten auch peu à peu von unten entwickelt werden - gerade auf Ebene der Fachgesellschaften. So sind in einigen Ländern die UEMS-Examina doch zu Standards für die Facharztprüfungen avanciert. Die UEMS wirbt seit Jahren bei Nationalen Stellen um derartige Anerkennung.

Allen voran die Prüfung des European Boards of Anesthesiology (EBA, [8]). Österreich, Malta und die Niederlande haben Teile dieses UEMS-Examens in Anästhesiologie als Grundlage für eigene nationale Facharztprüfungen genommen oder erkennen es als gleichwertig an. Auch Ungarn, Bulgarien, Polen greifen auf Teile zurück. Ein Prozess Bottom-up, von unten nach oben, der am Ende zu länderübergreifenden Standards führen könnte. „Die Anästhesisten sind da viel weiter als die Orthopäden“, lobt auch Grifka. Selbst hierzulande forderten Stellenanzeigen für Narkoseärzte zunehmend das UEMS-Examen ein.

Die Arbeitgeber könnten es richten. Je häufiger vielleicht auch das EBOT-Examen in Stellenanzeigen auftaucht, desto reger dürfte die Teilnahme daran werden. Grifka: „So etwas muss eben auch unterstützt werden.“

\section{Bernhard Epping}

\section{Literatur}

1 www.efort.org/education/efort-whitebook/

2 www.uems.eu/about-us/medical-specialties

3 www.uemssurg.org/section-and-board/ ebsq-examination

4 www.ebotexam.org/

5 www.uems-ortho.org/

6 Mäkinen TJ, Madanat R, Kallio P et al. The current state of the fellowship examination of the European Board of Orthopaedics and Traumatology (EBOT). Eur Orthop Traumatol 2014; 5: 217-220

7 www.anerkennung-in-deutschland.de/ html/de/statistik_zum_bundesgesetz.php

8 www.esahq.org/education/edaic/about

9 www.efort.org/about-us/vision-and-mission/

10 www.uems.eu/

11 www.cpme.eu

12 www.uemo.eu

13 www.aemh.org/pages/home.html

\section{Informationen für Ärzte aus dem Ausland, die in Deutsch- land arbeiten möchten}

Bei der EU-Kommission:

ec.europa.eu / internal_market / qualifications / directive_in_practice / automatic_recognition/doctors / index_de.htm

Beim BMBF:

www.bmbf.de/de/15644.php

www.anerkennung-in-deutschland.de/ html/de/

Broschüre des Bundesamts für Migration und Flüchtlinge:

www.daserste.de/information/wirtschaft-boerse/plusminus/sendung/ hr/2013/berufszugang-100.pdf

Marburger Bund:

www.marburger-bund.de/projekte/ auslaendische-aerzte-foreign-physicians/deutsch

Stand der Links 29.04.2015 